\title{
Light Curves of Lucy Targets: Leucus and Polymele
}

\author{
Marc W. Buie ${ }^{1}$ (1), Amanda M. Zangari ${ }^{1}$, Simone Marchi ${ }^{1}$, Harold F. Levison ${ }^{1}$, and Stefano Mottola ${ }^{2}$ \\ Southwest Research Institute 1050 Walnut Street, Suite 300 Boulder, CO 80302, USA; buie@ boulder.swri.edu \\ ${ }^{2}$ DLR - German Aerospace Center, Berlin, Germany \\ Received 2017 March 26; revised 2018 February 15; accepted 2018 March 26; published 2018 May 18
}

\begin{abstract}
We present new observations from 2016 of two Jupiter Trojan asteroids that are targets for the Lucy Discovery mission. The extremely long rotation period of (11351) Leucus is confirmed and refined to a secure value of $445.732 \pm 0.021 \mathrm{hr}$ with photometric parameters of $H_{r}=11.046 \pm 0.003$ and $G_{r}=0.58 \pm 0.02$ in the SDSS $r^{\prime}$ filter. This leads to a geometric albedo of $p_{V}=4.7 \%$. The amplitude of the light curve was measured to be 0.61 mag, unchanged from the value of one-fourth of a revolution earlier, suggesting a low obliquity. The first lightcurve observations for (15094) Polymele are also presented. This object is revealed to have a much shorter rotation period of $5.8607 \pm 0.0005 \mathrm{hr}$ with a very low amplitude of 0.09 mag. Its photometric parameters are $H_{r}=11.691 \pm 0.002$ and $G_{r}=0.22 \pm 0.02$. These values lead to a refined geometric albedo of $p_{V}=7.3 \%$. This object is either nearly spherical or was being viewed nearly pole-on in 2016. Further observations are required to fully determine the spin pole orientation and convex-hull shapes.
\end{abstract}

Key words: minor planets, asteroids: individual (Leucus, Polymele)

Supporting material: data behind figures

\section{Introduction}

Two groups of asteroids flank Jupiter in its orbit. These objects, known as Trojan Asteroids, orbit at Jupiter's L4 and L5 Lagrange points, sharing its semimajor axis of $5.2 \mathrm{au}$ (Barucci et al. 2002). As of late 2017, there were more than 4000 asteroids known in the leading L4 cloud and nearly 2500 in the trailing L5 cloud. ${ }^{3}$ Yoshida \& Nakamura (2005) estimate that the number of Trojans greater than $1 \mathrm{~km}$ is comparable to the number of objects in the asteroid belt.

Lucy is a mission recently selected in NASA's Discovery program, with a planned launch in 2021. It will perform six flybys: one main-belt asteroid and five Jupiter Trojans, one of which is a binary. For the first Trojan flyby mission, the object list is curated to include objects in both swarms, binaries and non, and cover the range of common Trojan spectral types. Among these objects are (15094) Polymele-formerly 1999 $\mathrm{WB}_{2}$ or $1997 \mathrm{WR}_{57}{ }^{4}$ — with its flyby slated for September 2027 and (11351) Leucus-formerly $1997 \mathrm{TS}_{25}$ or $1996 \mathrm{VP}_{36}{ }^{5}$ with a flyby slated for April 2028. Both asteroids are members of the L4 swarm (Levison \& Lucy Science Team 2016).

Previously for Leucus, French et al. (2013) found an extraordinarily long rotation period of $513.7 \pm 1.3 \mathrm{hr}$ (about 21 days). Such long periods appear to be overly common among Jupiter Trojans (French et al. 2015; Szabó et al. 2017). The French et al. (2013) period is based on observations spanning 34 days from 2013 March 29 to 2013 May 1, fewer than two rotations. There is a large per-night scatter, and the light curve appears quite asymmetric. Its primary minimum appears to be about 1 mag deep, but its secondary minimum is only about 0.6 deep. Both maxima are about the same. Using data from the Wide-field Infrared Survey Explorer (WISE), Grav et al. (2012) estimate an albedo of

\footnotetext{
3 http://www.minorplanetcenter.net/iau/lists/t_jupitertrojans.html

4 http://www.minorplanetcenter.net/db_search/show_object?object $\mathrm{id}=15094$

5 http://www.minorplanetcenter.net/db_search/show_object?object_ $\mathrm{id}=11351$
}

$0.079 \pm 0.013$, and a diameter of $34.16 \pm 0.65 \mathrm{~km}$ from an $H$-magnitude of 10.7. Using IRAS, Tedesco et al. (2002) find an albedo of $0.063 \pm 0.014$ and a diameter of $42 \pm 4$ $\mathrm{km}$. Levison \& Lucy Science Team (2016) list it as a D-type asteroid, one of the most common among the Trojans (Barucci et al. 2002). Vereš et al. (2015) estimate "mostlikely" $H$ and $G$ parameters for Leucus (and nearly a quarter of a million other asteroids) using Monte Carlo techniques. Using the methodology of Bowell et al. (1989), they find $H=11.37$ and $G=0.25$. Using the methodology of Muinonen et al. (2010), they find $H=11.18$ and $G=$ 0.21 . Hainaut et al. (2012) include colors for Leucus in the Minor Bodies in the Outer Solar System (MBOSSes) database, reporting $B-V=0.739 \pm 0.044, \quad V-R=$ $0.498 \pm 0.044$, and $R-I=0.402 \pm 0.072$.

Less is known about (15094) Polymele. Levison \& Lucy Science Team (2016) list it as a P-type asteroid. P-type asteroids are a sub-class of the reddish X-type asteroids that have low geometric albedos (Bowell et al. 1978; Tholen 1984; Zellner et al. 1985; Bus \& Binzel 2002; Clark et al. 2003). P-type asteroids are less common in the leading cloud than D-types (Grav et al. 2012). Using WISE data, Grav et al. (2012) estimate an albedo of $0.091 \pm 0.017$, and a diameter of $21.08 \pm 0.14 \mathrm{~km}$ from an $H$-magnitude of 11.6 . There are no known light-curve observations. Polymele was also included in the $H$ and $G$ predictions of Vereš et al. (2015). They find $H=11.56$ and $G=-0.26$ using the methodology of Bowell et al. (1989), and $H=12.04$ and $G=0.86$ with the methodology of Muinonen et al. (2010). Hainaut et al. (2012) include colors for Polymele in the MBOSSes database, reporting $B-V=0.652 \pm 0.065, V-R=0.477 \pm 0.065$, and $R-I=0.322 \pm 0.094$.

As an aid to the Lucy mission, we present detailed photometry on these objects. For Leucus, we further refine the period and reconcile our results with the French et al. (2013) data set. For Polymele, we provide the first light curve and period fits. For each object, we provide a solar phase curve and a new estimate of the albedo. 
Table 1

Summary of Observations

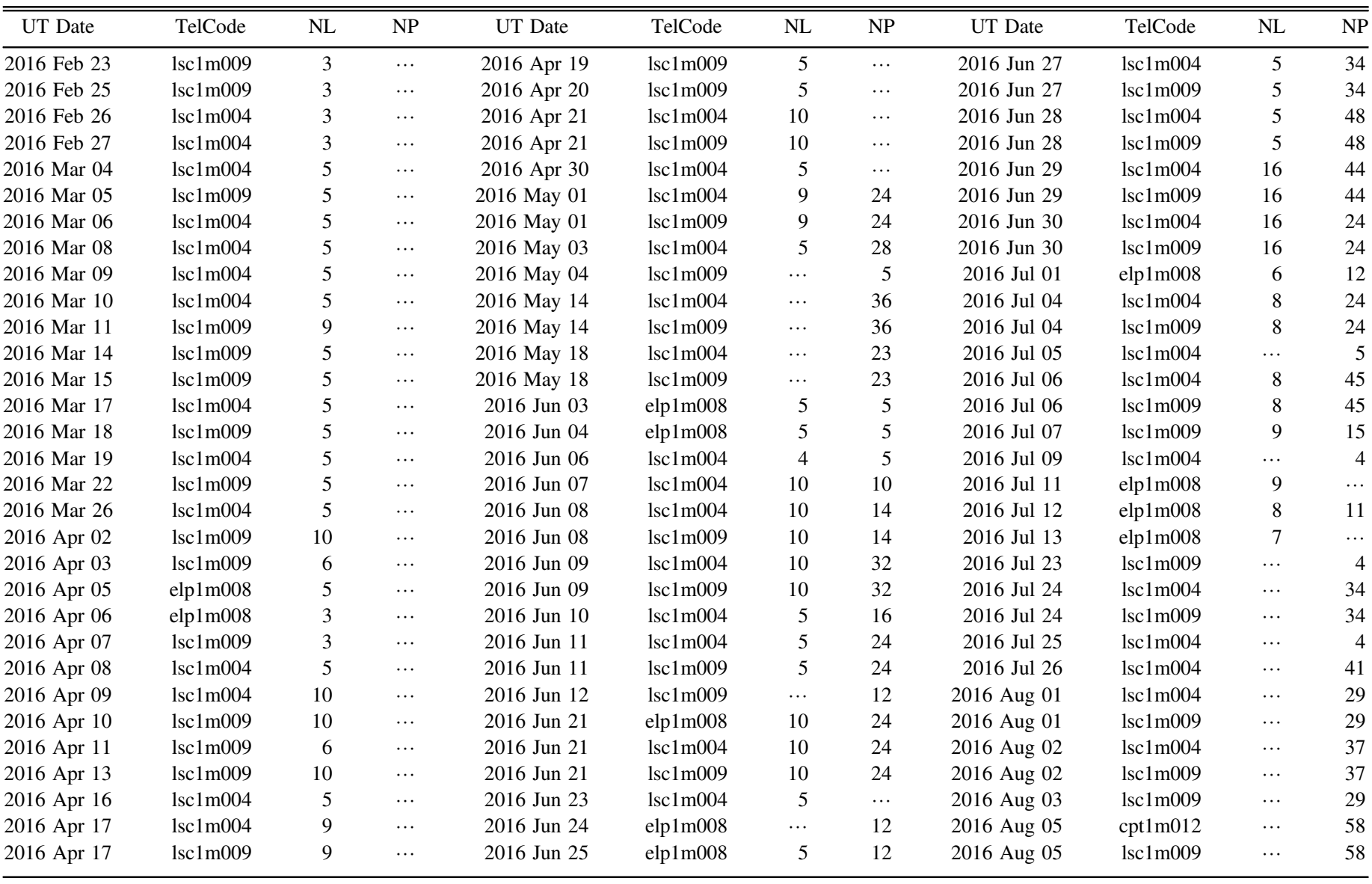

Note. NL is the number of images taken of Leucus. NP is the number of images taken of Polymele. TelCode starts with the telescope code: 1sc-Cerro Tololo, elpMacDonald Observatory, and cpt-South African Observatory, Sutherland and ends with the camera code.

\section{Observations}

All observations for this work were obtained from the Las Cumbres Global Telescope Network (LCOGT; Brown et al. 2013). We used the new Sinistro cameras - then deployed only on two telescopes at Cerro Tololo (MPC codes W86 and W87), and on one telescope at MacDonald Observatory (MPC code V37). At the very end of this work, a new camera was commissioned at Sutherland (MPC code K93) and data were collected there on one night. The systems are nearly identical, differing only in sky-plane orientation.

The Sinistro cameras have a $4096 \times 4096$ pixel detector with a image scale of $0.390 \mathrm{arcsec} / \mathrm{pixel}$, giving a $27 \mathrm{arcmin}$ square field of view. The read noise is $12 \mathrm{e}-/ \mathrm{DN}$ and dark current is less than $1 \mathrm{DN}$ per pixel for our integration times. All observations were taken with an SDSS $r^{\prime}$ filter. Most data were taken with 300-second exposures, but the last lunation of data on Polymele used $360 \mathrm{~s}$ to compensate for the increased phase angle and greater target distance. Mean seeing at MacDonald was 2.7 arcsec, with most nights falling between 2 and 3.3 arcsec. Seeing at Cerro Tololo ranged from 1.4 to 3 arcsec; the seeing distribution was tri-modal with peaks at $1.7,2.1$, and 2.4 arcsec (in descending order of occurrence). Most observations were collected at Cerro Tololo due to the southerly declinations of the objects.

Observations at LCOGT are handled by a robotic scheduling system with the intent of seamless scheduling across its global network of observatories. A summary of observations is given in Table 1, where the number of images taken on a given UT date and telescope are tallied by object. During the time of our project, however, systems with the camera we needed were at very similar longitudes. The observable window at the MacDonald site was significantly shorter than for Cerro Tololo. As a consequence, we were forced to build our observation requests carefully considering the observing window from Cerro Tololo.

The observations for Leucus began on 2016 February 22, as soon as the observing window was long enough to be useful and ran until 2016 July 13 . We already knew that the rotation period was long, and this led to our strategy of collecting a small number of observations per day. While the observing window was short, we limited the requests to one block per day. Later, when the observing window became longer than $6 \mathrm{hr}$, we added a second daily block separated by at least $4 \mathrm{hr}$. As can be seen in Table 1, the very first blocks consisted of just three images, but this turned out to be insufficient for the data reduction requirements. We later standardized on five frames per epoch. The nights of June 29 and 30 were an exception: more observations were requested near the time of minimum light in an attempt to look for evidence of binarity.

All images were taken with the SDSS $r^{\prime}$ filter to provide the highest throughput available from the LCOGT filter set. We did not attempt to get additional color observations. Our chosen 

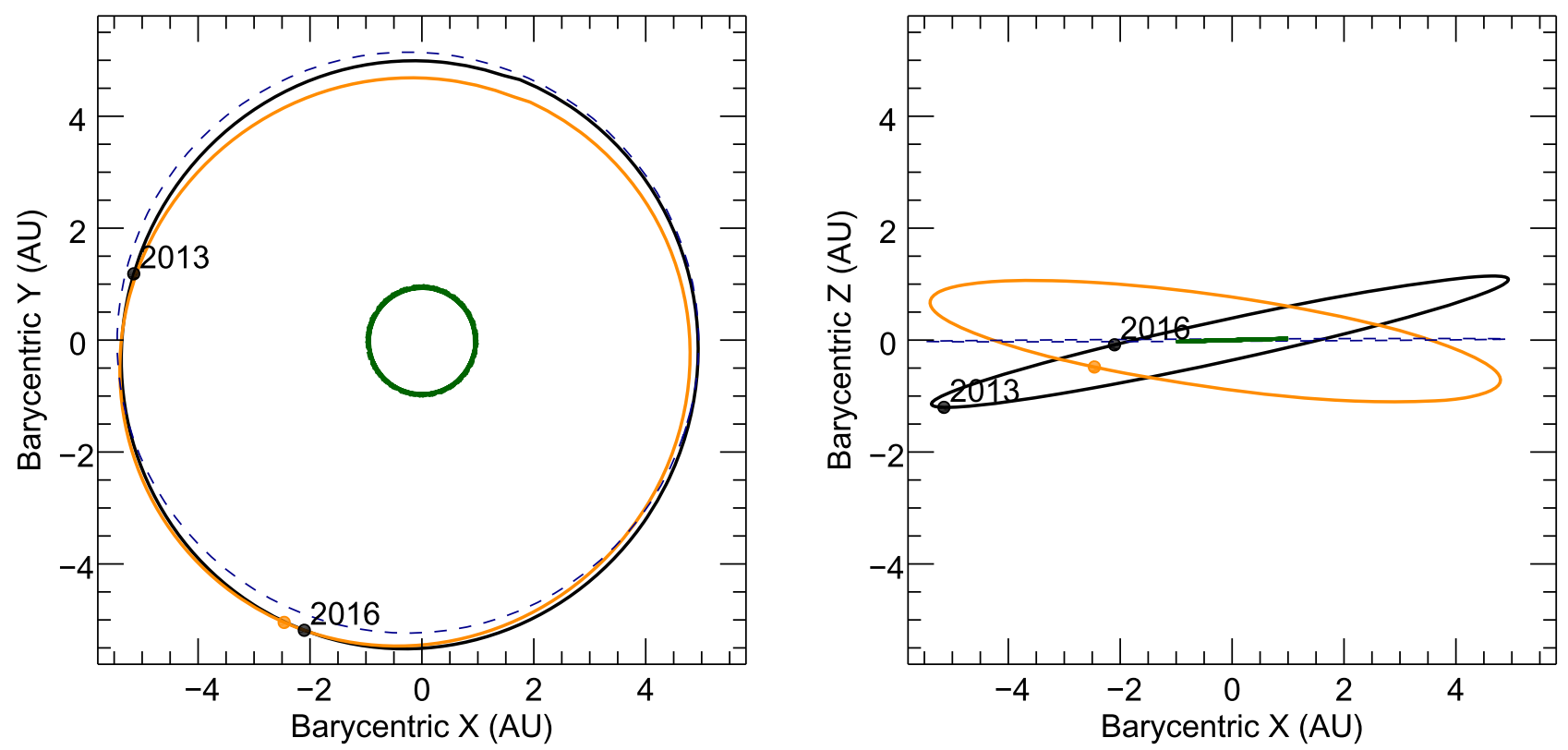

Figure 1. Orbit and viewing geometry for Leucus and Polymele. The green circle shows the position of the Earth and the dashed line is the position of Jupiter over 12 years. The solid curves are for Leucus (black) and Polymele (orange). The symbols indicate epochs of light-curve observations, twice for Leucus and once for Polymele.

filter also happens to be supported by the APASS photometric catalog (Henden et al. 2012). All images were set up with nonsidereal tracking to follow the motion of the object. The exposure time was set to maximize the signal on the object while minimizing the trailing of the field stars. We would have preferred to track at halfway between the rate of the object and sidereal to equalize smearing between Trojan and stellar background, but this option was not supported by LCOGT. At opposition, the mean motion on the sky peaks at 10 arcsec/hour, meaning the stars are trailed by 0.8 arcsec within a single exposure.

As shown in Table 1, the observations for Polymele began on 2016 May 01 and ran until 2016 August 05. We had no prior knowledge of the rotation period and started with an observing cadence with much longer sequences of images per night in anticipation of a short rotation period. We would have preferred longer exposure times than for Leucus, but to do so would have increased the photometric effects of smearing and compromised the data. The observations were usually requested in batches of 12 observations to fall within an hour, though those images do not fill an hour time slot. At the time these observations were taken, there seemed to be a bias in the scheduling system against longer, uninterrupted blocks of observations and shorter segments seemed to be more successful. Unfortunately, this introduced time gaps between segments, but there were no controls to prevent this. These gaps led to more severe aliasing during early period searches as the data were coming in. There were enough data in the final data set to overcome the problems caused by the gaps.

The viewing geometries for both objects are summarized in Figure 1. The orbits for Leucus (black) and Polymele (orange) are shown to scale along with the orbits for Jupiter (blue dash) and the Earth (green). The position of Leucus is marked for the French et al. (2013) observations in 2013 and our new observations in 2016. The position of Polymele is marked for the 2016 observations - the only ones we have available. Leucus moved one-fourth of the way around in its orbit between the two epochs, but there was also a significant out-of- plane motion component as well. The 2016 Leucus observations were also at very low ecliptic latitude, accounting for the low minimum phase angle we obtained.

\section{Data Reduction}

The level of smearing due to the LCOGT-imposed tracking option was not expected to significantly impact the photometry, but it was the primary reason we chose not to use PSF fitting for the photometric extraction. Instead, all photometry was generated with synthetic aperture summation as described in Buie \& Bus (1992). In the case of these data, two additional alterations to that methodology were introduced. The initial guess for the placement of the photometric aperture was derived from the ephemeris of the object to be measured, once converted to an $(x, y)$ pixel position via an astrometric solution for the image. The second improvement in the processing was to subtract a background image from the data prior to the measurement of the object flux.

LCOGT delivers its images after applying bias, dark, and flat fielding corrections. Once we had their pipeline processed data, the images were processed with the following sequence of operations. The IDL routine used from either Buie's IDL Library $^{6}$ or the Astronomy User's Library is noted.

1. Estimate the seeing (seeing).

2. Extract a list of positions and instrumental fluxes (findsrc).

3. Generate an average PSF (psfstack).

4. Generate an astrometric solution (astrom).

5. Determine photometric transformation (srcor, robomean).

6. Build a reference image for the image (warpstack: calls dewarp, avgclip).

7. Subtract reference image (ois).

6 http://www.boulder.swri.edu/ buie/idl 


\section{Extract final photometry from difference image (basphote).}

The seeing estimate generated in step 1 was used to set the object aperture radius $(O)$ equal to the seeing value (FWHM in pixels) rounded up to the nearest integer and could vary from image to image. A list of sources on each image was built by scanning for pixels that exceed the sky background by at least $5 \sigma$ and less than a saturation limit set to 75,000 counts. The routine that does this requires a characteristic scale for the image, and we used the object aperture radius from the previous step. The inner and outer radius of the sky annulus for the synthetic aperture photometry at this step were set to $O+1$ and $O+5$. Stars from this list were used to build an average numerical PSF image from a 501 pixel region centered on the asteroid. This step was not strictly necessary but made it easier to identify and exclude images where the automated focus system failed. It also served as a useful image quality check.

The astrometric solution for each image was an absolutely essential component of our data processing. The automated LCOGT pipeline attempts to provide a WCS solution in the header but this fails often enough to be useless for our needs. In the end, we never used their WCS and just used the headerprovided telescope position to drive the astrometric reductions with our tools. Our astrometry tool was able to run automatically by correlating the source list with a sub-catalog using reference stars from the UCAC4 catalog (Zacharias et al. 2013), corrected for proper motion to the epoch of the image. A third-order polynomial fit was derived for the mapping of pixels to and from tangent plane coordinates. For convenience, this solution is saved back to the header of the image to be used by later steps. The astrometric system used is described more in Buie et al. (2018).

Photometric transformation was derived from on-chip sources from the version 9 APASS catalog (Henden et al. 2012). The APASS catalog position was used to correlate with our source list and those matching within 3 arcsec were kept. The correlated catalog list was filtered down to eliminate sources with $\mathrm{S} / \mathrm{N}<50,\left(g^{\prime}-r^{\prime}\right)>2.5$, and $r^{\prime}>30$. This list of matched and filtered sources is then remeasured with the same object aperture as before but with a much larger sky aperture $(O+5$ to $O+100)$ to get the instrumental magnitudes for calibration. We see no evidence for any color term for the transformation from instrumental to standard magnitudes and thus just needed to determine a photometric zero-point. The surviving list was then processed with a series of robust mean determinations. At each step, there was a list of flags for each correlated star that indicated if it was good or bad. All sources started as good, but each test had the opportunity to mark additional objects as bad. In order, the following robust means were computed: mean difference between APASS and measured R.A. and then decl., mean magnitude difference between instrumental and standard magnitude, and the mean difference between instrumental and standard magnitudes in units of a standard deviation where the measured and catalog errors were combined to obtain a standard deviation for each source. These calculations were used only to filter out measurements inconsistent with the distribution at greater than $3 \sigma$. The final step was to compute a weighted mean of the instrumental minus standard magnitude of those sources that were still considered good at the end of the filtering. The mean

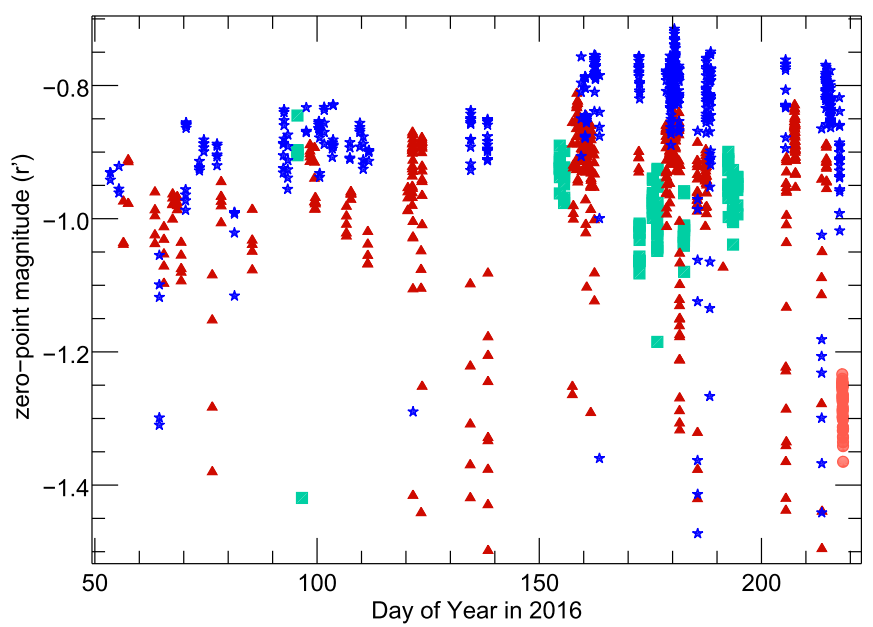

Figure 2. Photometric zero-points for all observations. Color and shape denote different telescopes: orange circles - Sutherland (cpt1m012), green squaresMcDonald (elp1m008), red triangles-Cerro Tololo Dome B (1sc1m004), and blue stars-Cerro Tololo Dome C (1sc1m009).

and the standard deviation of the mean then determined the photometric transformation to standard SDSS $r^{\prime}$ magnitudes.

Figure 2 shows a graphical summary of the per-frame photometric zero-points. For each telescope/instrument combination, the upper bound of the plotted points is indicative of the throughput of the system. The sparser points that plot lower were observed under conditions with thin clouds. The larger timescale pattern (peaking around DOY 180) is a general consequence of the targets being at opposition in the middle of the year, leading to better observing circumstances.

Due to the crowded field, we needed to remove the stellar background to avoid photometric contamination. Some images did not need correction, but we applied the same processing to all data. For each image, a 501 pixel square region of interest (ROI) was centered on the ephemeris location of the target. Every other image we took was a candidate for the reference image template-including data taken on other telescope and instrument combinations at LCOGT. For an image to be considered for the stack, it had to overlap the ROI by at least $65 \%$ and the target position in the reference image candidate had to be at least 10 arcsec away from the target position on the measurement image.

Each image in this set was resampled to exactly match the sampling of the ROI of the image to be processed. The resampling was driven by and critically depended on the quality of the astrometric solution for the image and all of the candidates for the reference image stack. This resampling and stacking was done for each reference image to prevent resampling the original data. This procedure ensured the photometric zero-point still applied once the background template was subtracted.

The stacking software also used the photometric zero-point and measured FWHM of all images as well as the sky background and its noise from each ROI. This ancillary information was used to grade the value of each reference image's contribution to the stack to automatically construct the best stack given the candidate reference images. If the seeing in the set of reference images varied by more than $25 \%$, the worst quartile of the frames by seeing was discarded. The quartile was defined by range of seeing seen, not the sorted number by seeing; thus, this filtering step could remove just one image, if 
it were a singular outlier. If there was a more diverse distribution of seeing, the number of frames removed was higher. A figure-of-merit (FOM) was computed from the difference of the seeing from the mean divided by the mean seeing. The images were then sorted by this FOM for subsequent processing.

The reference image with the highest transparency, measured by the maximum zero-point value, was used for the flux reference image. The zero-point was converted to a flux reference value (absolute scaling not important) for each image. Each image was scaled to match the flux reference value of the reference image. An estimate was then computed of the noise level and limiting magnitude of the stack before and after adding each new image given all the prior images that were considered acceptable. If adding an image would have improved the final $\mathrm{S} / \mathrm{N}$ of the stack, it was kept. Those that would have degraded the stack were discarded. Generally, the best image quality data were kept and the worst data were discarded, but the interplay between sky background level (variable moonlight), transparency (photometric zero-point), and seeing all had different effects on the value each image brought to the reference stack.

Once the set of scaled reference images was evaluated, the good images were then averaged with outlier rejection. For each pixel in the stack, the highest $25 \%$ of the pixel values were suppressed in the initial estimation of the noise level in that pixel. Those that exceeded the mean by more than $3 \sigma$ were rejected from the final mean. The mean and the standard deviation in each pixel evolved as points were removed until the final result converged when no more pixels were marked as bad. Operationally, this process worked very well when there were at least 10 images from which to build a template. This was not a rigorous goal, but in our case we often had many more. The minimum number of frames in a template was 3 and the maximum was 56 for Leucus and 164 for Polymele. The reference image and thus the photometry is better with more images contributing to a template but the measurement uncertainties track the actual number used.

The background template image has essentially perfect spatial registration relative to the data image but the image quality and flux scaling are different. We used the optimal image subtraction (OIS) algorithm described by Alard \& Lupton (1998) to generate the difference image. Their original software was ported to IDL and described by Miller et al. (2008). The version in the Buie IDL library was optimized for fast execution by Buie and an undergraduate team at the Harvey Mudd College. Our version differed from the original only in its handling of the basis set for the PSF-matching convolution. The original used a set of overlapping Gaussians, while we used the delta-function basis set introduced by Miller et al. (2008). The OIS process requires fitting and removing a variable sky level from both image and template and then flux scaling the reference to match the data. After subtraction, the mean sky level in the data is added back to retain the original sky and noise characteristics of the original data. At this point, any gradient in the sky as well as the background sources have been removed from the ROI image.

With the difference image in hand, it was then a simple matter to extract synthetic aperture photometry on the object using the same apertures as the reference stars for the zeropoint determination. Adding the zero-point and propagating errors yielded the final absolute flux measurement of the source. An example image is shown in Figure 3. The data image shown has had no processing other than typical steps such as flat fielding and bias subtraction. The small red circle is centered on Polymele and shows the photometric aperture with a radius equal to the FWHM. The width of this sub-image is 195 arcsec across. The center panel is a reference image built in the manner described above. This example had 133 images contributing to the average. The small red circle is plotted at exactly the same location as in the data image. The template image clearly goes much deeper. The final panel on the right shows the result of subtracting the template image from the data. The sky is very clean, as is the area near Polymele. The brightest stars leave behind poorly subtracted regions but none of these fell on the object and their presence in the sky annulus did not affect the measurement of the sky level. This process served to extract photometry that is largely limited by the number of detected photons, rather than noise from sky background, stellar background, or detector noise.

\section{Light-curve Analysis}

The processing of the photometry was very similar for each object. The apparent magnitudes were corrected for heliocentric and geocentric distances and phase angle to a standard absolute magnitude for unit distance and zero degrees phase. We used a guess for $G$ in the initial stages, but in the final analysis the value of $G$ was adjusted to minimize scatter in the final phased light curve. When adjusting the phase coefficient, the period was always checked to get a converged value. For our final data set, there was no correlation between period and $G$.

We scanned for a period used a variant of the technique described in Buie \& Bus (1992), which is a phase-dispersion minimization tool. These data suffered somewhat from sparse sampling or aliasing at some trial periods. The usual phase binning approach did not always work well for some subsets of the data. A trivial modification to the PDM technique was made to perform an $n$ th-order Fourier-series fit at each trial period and then save the $\chi^{2}$ values. For both objects, a second-order fit was sufficient. We found this method to be just as fast but much more stable in the presence of sparse or aliased data than the older phase binning technique.

The period search employed a two-part scan. First, a course scan over a very wide range of periods was run. The range of periods searched was tuned for each object but used 5000 steps over the range searched. In this range, the trial period with the minimum $\chi^{2}$ value was used as the center of a narrow scan. The width of the narrow scan covered 2000 trial periods centered on the best period from the first step. The following sub-sections gives the actual settings used and the results obtained.

\subsection{Leucus}

The final data set includes 378 observations taken on 60 distinct dates. Of these, 360 observations were deemed useful and are reported here. Figure 4 shows a large-range period scan running from 200 to $700 \mathrm{hr}$. This range includes the single $\min / \max$ rotation period as well as the value suggested by French et al. (2013). Strong minima occur at two periods, one at half the value of the other. The French et al. (2013) period of $513 \pm 1.3 \mathrm{hr}$ is strictly excluded by these data. The lowest local minimum corresponds to the double min/max light curve 

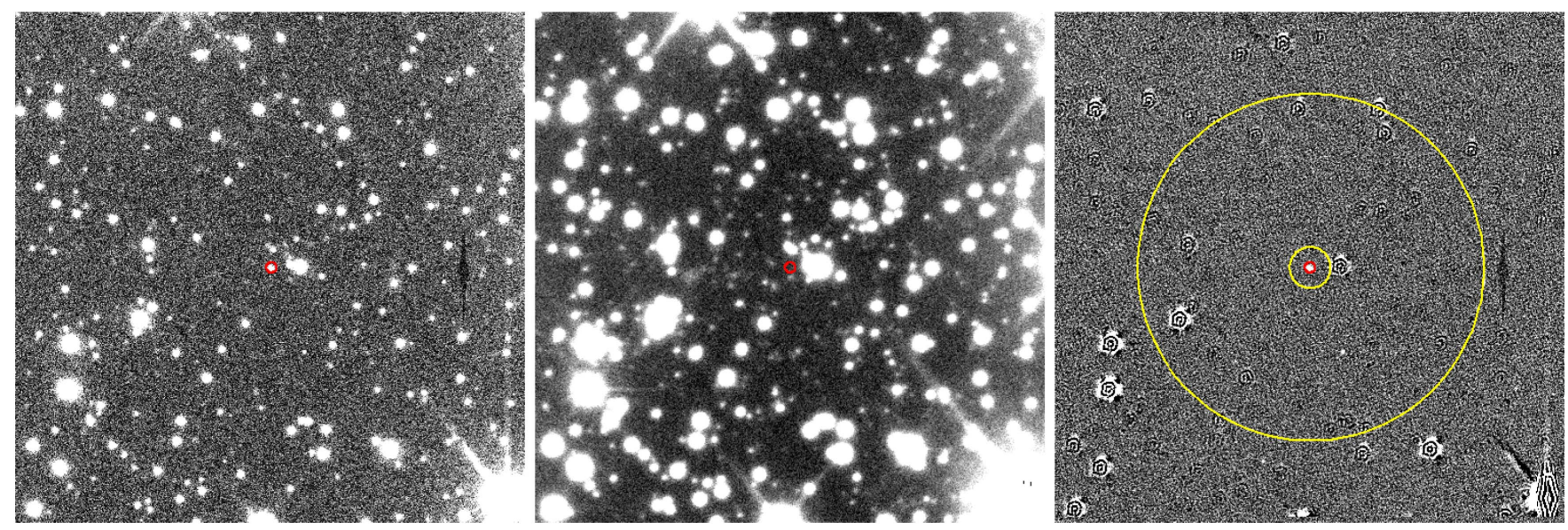

Figure 3. Example of typical image. The left panel is a $501 \times 501$ pixel sub-array centered on Polymele for image cpt1m012-f106-20160805-0071-e91. The center panel is the template image constructed from 133 other images that contain the same region of the sky as the data image. The right panel shows the result of the optimal image subtraction where the background field has now been removed. The red circle shows the object aperture used on this frame. The two larger yellow circles show the sky annulus used for sky subtraction.

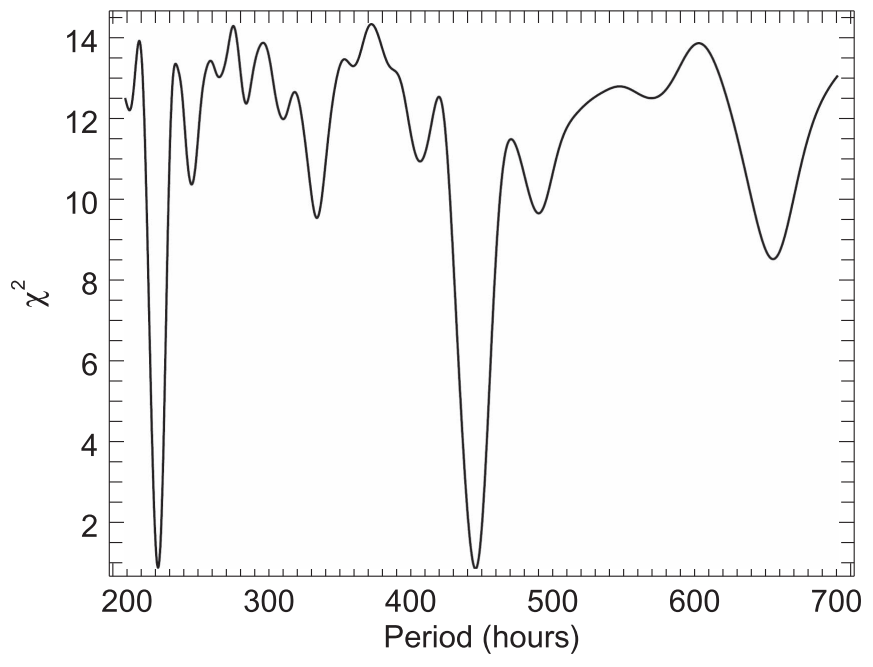

Figure 4. Period scan for Leucus using data from 2016.

at a period of $223.07 \mathrm{hr}$ and $\chi^{2}=1.032$, which is $11.5 \sigma$ away from the best period.

The final period comes from a very narrow scan was set to $\pm 0.4 \mathrm{hr}$ about the best period shown in Figure 4. In the narrow scan, the $\chi^{2}$ minimum is very well-behaved and allowed for a good estimate of the uncertainty on the period.

The period based on just our 2016 data is $445.81 \pm 0.24 \mathrm{hr}$. The data were taken over a 141-day range, covering 7.6 rotations. The phase angle ranged from $0^{\circ} .125$ to $10^{\circ} .2$, and we determined a phase parameter value of $G_{r}=0.58 \pm 0.02$. The mean absolute magnitude is $H_{r}=11.046 \pm 0.003$. The Fourier-series light-curve fit coefficients are given in Table 2 . The amplitude of the light curve measured strictly from the minimum and maximum of the fitted curve is $0.61 \mathrm{mag}$. The value of $\chi^{2}$ given in the table is from a weighted fit of the curve to the data using the measurement uncertainties. The goodness of fit clearly indicates the fit is not perfect or the uncertainties are underestimated. We used a conservative assumption that the uncertainties are too small and applied an aposteriori adjustment to give $\chi^{2}=1$. The uncertainties on all derived photometric properties shown here are computed after this aposteriori adjustment.
Table 2

Light-curve Coefficients for Leucus

\begin{tabular}{ccccc}
\hline \hline$n$ & $a_{n}$ & $\sigma_{a}$ & $b_{n}$ & $\sigma_{b}$ \\
\hline 0 & 11.046 & 0.003 & $\ldots$ & $\ldots$ \\
1 & -0.023 & 0.004 & -0.011 & 0.005 \\
2 & -0.290 & 0.005 & -0.019 & 0.004 \\
\hline \multicolumn{5}{c}{$|O-C|=0.05 \mathrm{mag}$} \\
& \multicolumn{1}{c}{$\chi^{2}=19.8$} \\
\hline
\end{tabular}

Note. Parameters computed for a period of $445.732 \mathrm{hr}$ and a phase coefficient of $G=0.58$. All observations taken with a SDSS $r^{\prime}$ filter. These values are based on the 2016 light-curve photometry with the period from the combined data set.

The analysis of French et al. (2013) from their 2013 data suggested a period of $513.7 \pm 1.3 \mathrm{hr}$ with a possible secondary period at $53.28 \pm 0.04 \mathrm{hr}$. However, these data only covered 33 days or 1.8 rotations using our new period (1.5 rotations for their period). Slow rotation periods can be very difficult to measure, requiring observations over a very large number of nights for proper sampling. When our more extensive data are phased using the French et al. (2013) period, there is no coherent light curve pattern at all. Our data completely rule out this longer rotation period. However, when phasing the French data with our period, a light-curve pattern is clearly evident. Figure 7 shows the French et al. (2013) observations phased with our period. The result shows a plausible match to our light curve, even with the same amplitude, but with a few caveats. For this plot, a phase shift of 0.27 rotations was applied to approximately account for the shift in viewing geometry. A $\left(V-r^{\prime}\right)$ shift of 0.38 mag was applied to line up the Fourierseries fit from our data with their data. The agreement between the two is excellent except for the data falling between phase 0.7 and 0.9. Looking closer, there are seven nights that stand out as clearly discrepant. At the same time, there are four other nights of their data near the same phase that do match our light curve quite well. If our period is correct, there is clearly an internal inconsistency in the French et al. (2013) data. Going forward, the red points are excluded from the rest of our analysis. 


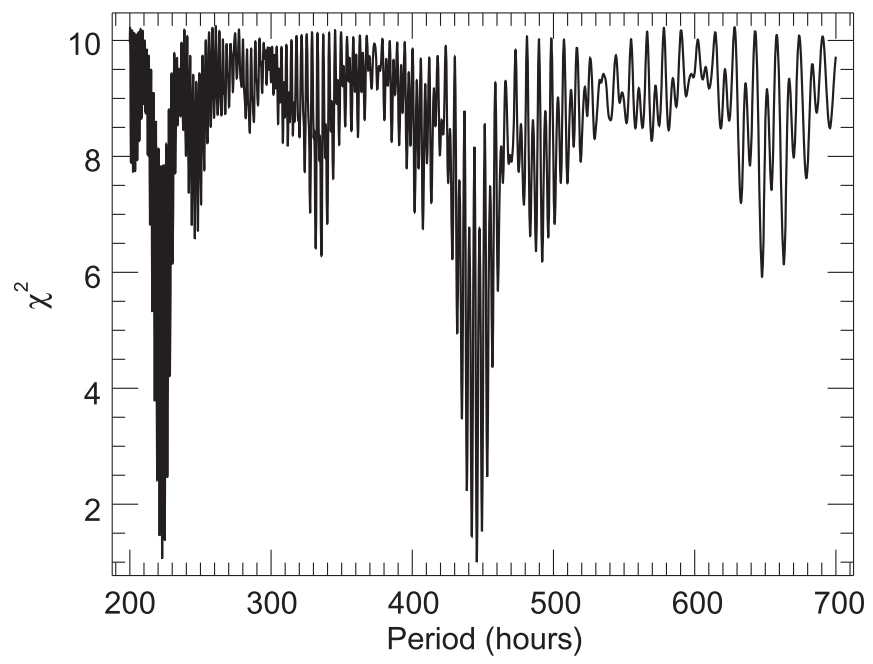

Figure 5. Period scan for Leucus using data taken in 2013 and 2016. A phase correction of 0.27 rotations was applied to align the 2013 and 2016 data to adjust for the different viewing geometry.

The inconsistent observations were taken between 2013 April 15 and 21, inclusive. A systematic shift of 0.18 mag for these nights makes all but one night agree quite well with the rest of the data, but we chose not to include these data in the period search. With those seven nights removed, we ran a period search to see if the rotation period could be further refined. Figure 5 shows the period scan from the combined data set. A phase correction of 0.27 (5.015 days) was applied to remove the geometric difference between the two epochs of data. For this result, the aposteriori uncertainty correction for the French data was 1.86. There are clear signatures in the period scan for periods that differ by one rotation from the best fit, but these alternatives have $\chi^{2}$ values significantly worse than the already excluded single $\mathrm{min} / \mathrm{max}$ light-curve option and clearly rule out all but one choice. The best period from the combined data comes out to $445.732 \pm 0.021 \mathrm{hr}$.

The 2016-only phased light-curve data are shown in Figure 6. The solid orange curve is the best-fit Fourier 2-term Fourier series to the data as provided in Table 2. In the electronic supplement, there is an associated data file for this figure that includes all the data as well as the original LCOGT data file name to facilitate correlation of our results with the contents of their data archive. The solid green curve is a scatterplot-smoothed curve that more closely follows the data without imposing a functional form. The data and curves are extended slightly on the left and right. The Fourier fit is a very close match to the trend curve but there appears to be a more sharper light-curve minimum than is tracked by the simpler fit and the amplitude of the smoothed curve is a little higher. Figure 7 shows the French et al. (2013) data phased with our best period. The curve overlain is the same as that plotted in Figure 6 and is an excellent match to the points plotted in black.

We were concerned about marking some nights of data from 2013 as bad without additional justification. We asked the investigators from French et al. (2013) to check their data, and they confirmed with a subsequent reanalysis that there were some calibration problems on these nights. It is clear now that those observations, coupled with the incorrect period, were responsible for the asymmetric and very low minimum in the phased light curve shown in French et al. (2013). Their longer period appeared to result in a sensible light curve only because the bad nights did not overlap with other nights of observation in their data set for the period they chose.

Our observations cover a wide range of solar phase angles, even reaching down to a minimum of 0.13 at opposition. The behavior with respect to phase angle is shown in Figure 8 . The phase coefficient is larger than was assumed by French et al. (2013) and has a significantly lower variation than a typical value of 0.15-0.2 (Bowell et al. 1978). No strong ultra-low angle surge was seen, but a departure weaker than $0.1 \mathrm{mag}$ cannot be ruled out. Also shown on this figure is a linear phase fit to the data. This fit has a slope of $0.032 \mathrm{mag} \mathrm{deg}^{-1}$ and an intercept of 11.109 with $\chi_{\nu}^{2}=1.35$. Based on the goodness of fit for the two cases, the linear case is $100 \sigma$ away from the $H, G$ fit according to the confidence intervals for the $H, G$ fit. Based on the formal errors, there is evidence for curvature in the phase behavior but there are clearly non-random deviations from the curve in the data. Further observations will be useful to confirm these results.

\subsection{Polymele}

The final data set includes 595 observations taken on 36 distinct dates. Only those data that were deemed useful are reported. We include the original LCOGT data file name in the table to facilitate correlation of our results with the contents of their data archive.

The light-curve amplitude is low compared to the perobservation photometric uncertainty and required special care to extract a period. Also, the gaps in the data created some problems with aliasing depending on the data chosen for a period search. To get a sense of the valid period hidden within the data, we did searches on subsets of data collected by month or further broken down by chunks of data within a single lunation with a month. The exact grouping of data was not important; rather, we looked for a period that was always present when the data subset were of sufficient quality. Minima due to aliasing never repeated from one subset to the next. A consistent minimum showed up just short of $6 \mathrm{hr}$. The coarse scans covered from 0.6 to $10.5 \mathrm{hr}$ and the scan from the full data set is shown in Figure 9. There are a few deep local minima in the scans, but the second-best is $41 \sigma$ away from the best. All of the longer period minima show string signatures of aliasing due to the temporal sampling while also showing highly unlikely light-curve shapes.

The data cover a wide range of phase angles (1.2-9.7) and thus provide a good constraint on $G_{r}=0.22 \pm 0.02$. In fact, the phase variation is larger than that caused by rotation. The best period from our data is $5.8607 \pm 0.0005 \mathrm{hr}$, and the phased data are shown in Figure 10. The mean absolute magnitude is $H_{r}=11.691 \pm 0.002$. The Fourier-series lightcurve fit coefficients are given in Table 3 and plotted as the solid red curve. The amplitude of the light-curve measured strictly from the minimum and maximum of the fitted curve is 0.09 mag. Similar to the Leucus light-curve, the solid green line is a smoothed version of the light curve that is not constrained by any fitting function. The goodness of fit and deviations between the two curves clearly indicate that the fit is not perfect. The original value of $\chi^{2}$ given in the table is from a weighted fit of the curve assumption that the uncertainties are too small and applied an aposteriori adjustment to give $\chi^{2}=1$. The uncertainties on all derived photometric properties shown here are computed after the aposteriori adjustment. 


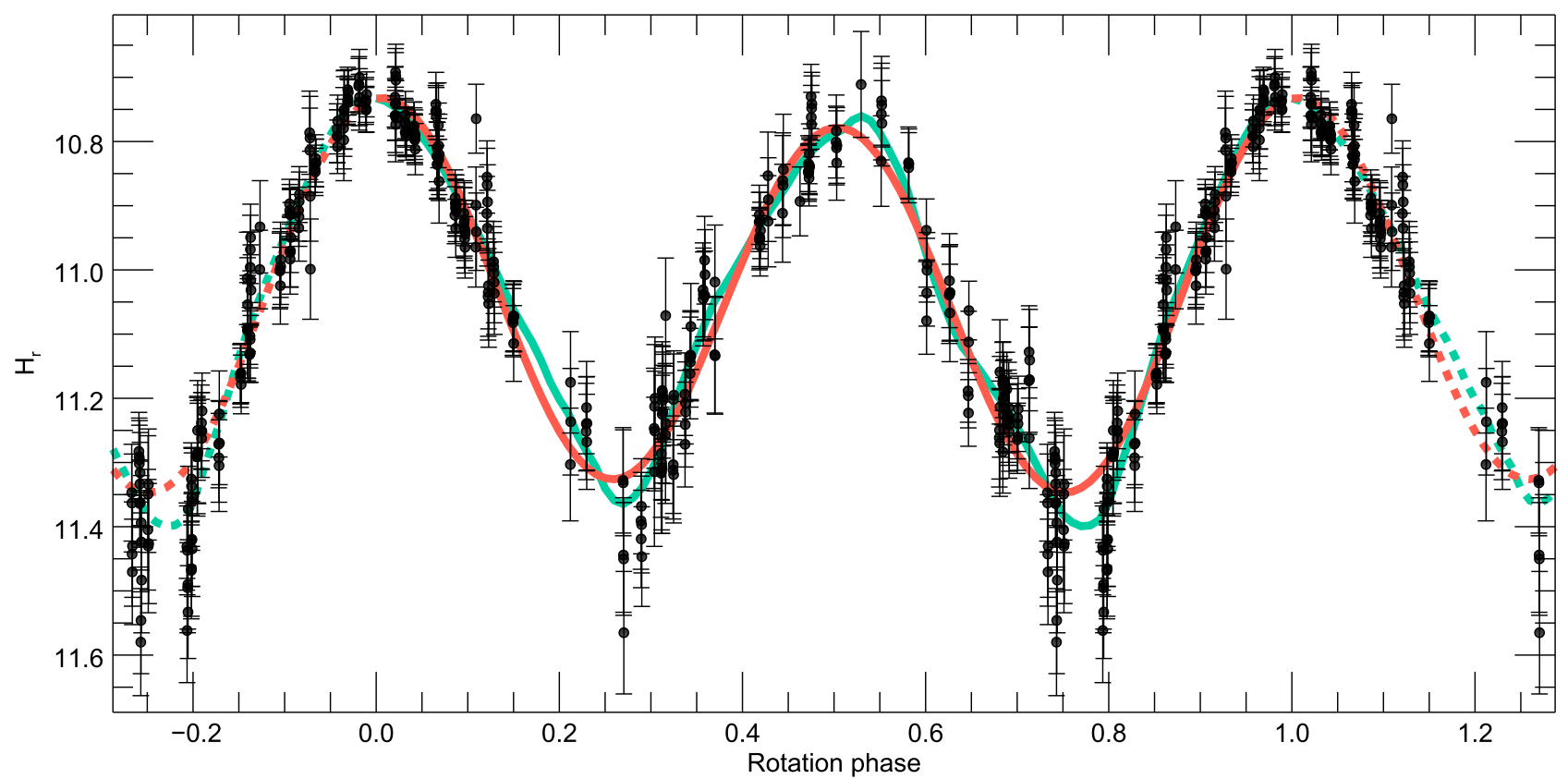

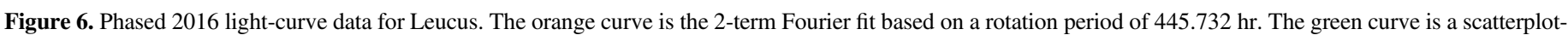
smoothed curve that more closely follows the data without imposing a functional form. The data used to create this figure are available.

Figure 11 shows the photometric phase behavior for Polymele. Distance effects and the rotational light curve have been removed. The dimming with increasing phase angle is nearly a factor of two larger than for Leucus, but this phase behavior is very typical of main-belt asteroids (Bowell et al. 1978). Unfortunately, we missed the lowest range of phase angles due to bad weather for weeks around the time of opposition. Also shown on this figure is a linear phase fit to the data. This fit has a slope of $0.050 \mathrm{mag} \mathrm{deg}^{-1}$ and an intercept of 11.822 with $\chi_{\nu}^{2}=0.9$. Formally, the linear fit is better than the standard $H, G$ fit to the data. Extending the data to an even lower phase angle will clearly be important for getting a more accurate absolute magnitude. Despite the better linear fit, we adopt the results of the $H, G$ fit for now.

\section{Discussion}

These new results permit an improved estimation of the albedos. Using the diameters from Grav et al. (2012), we obtained a $V$ geometric albedo of $4.7 \%$ for Leucus and $7.3 \%$ for Polymele. We did not have an accurate $\left(V-r^{\prime}\right)$ color for these objects, so we converted from $r^{\prime}$ to $V$ using a class-mean color. According to Ivezić et al. (2001), P-type objects have the same $\left(V-r^{\prime}\right)$ color as C-type asteroids, and D-type objects have the same color as S-type. These larger class color means are $\left(V-r^{\prime}\right)=0.18$ for C-type and $\left(V-r^{\prime}\right)=0.26$ for S-type objects. Our new result for Leucus indicates the albedo is even lower than previously estimated by Grav et al. (2012). This low albedo is certainly consistent with a primitive D-type object (Barucci et al. 2002). Our albedo for Polymele is only slightly lower than previous estimate. It is still a low-albedo object but clearly not as low as Leucus. The constraint on albedo is not as strong as it appears, though. The observations with WISE were collected on 2010 February 13. Our rotation period propagates back to 2010 with an uncertainty of $7 \%$ of a rotation. If we had no constraint on the period, the worst case albedo range we could infer is from $p_{v}=0.036$ to 0.062 . This value includes a rough estimate of 0.5 phase difference between the 2016 and 2010 data due to change in the ecliptic longitude of Leucus. Our estimate of the actual rotation phase is that the WISE data occurred at phase somewhere between 0.12 and 0.26 . This range excludes the bright end of the albedo range. Even with these considerations, the surface of Leucus has a very low albedo around $4 \%-5 \%$. Further work is required to properly combine the optical light-curve data with the WISE thermal data. Our size estimates are based on scaling the mean-light brightness to the WISE-measured diameter. Getting a more accurate period as well as determining the rotation pole is required for a better absolute size. For now, we can only qualitatively indicate the obliquity of Leucus is low.

The light-curve amplitude we measured for Polymele is very low. Given that we only have one epoch of data, we cannot make firm conclusions about shape or spin orientation. From the low amplitude, we can only say that we are either looking nearly pole-on to the object or it is nearly spherical in shape. Additional observations will be required to settle this question. Just one year's worth of change in aspect will provide some useful constraint.

Despite the low per-point $\mathrm{S} / \mathrm{N}$ for the Polymele data, the phased light curve is clearly significant. The lack of a singlepeak light-curve minimum in the PDM was of some concern, but the very weak light curve has relatively low power in the first Fourier component. When phased at half the period, the second-order component is strong enough to wash out the coherence from the first order component. No other period comes close to the statistical significance of our determination, and other minima also do not look reasonable for a phased shape-dominated light curve.

The light curve result for Leucus is very solid. The long rotation period makes this object particularly difficult, but the observing cadence permitted by robotic telescopes is extremely well suited to this task. Our data span many rotations within this year and give us a solid phased light curve that was 


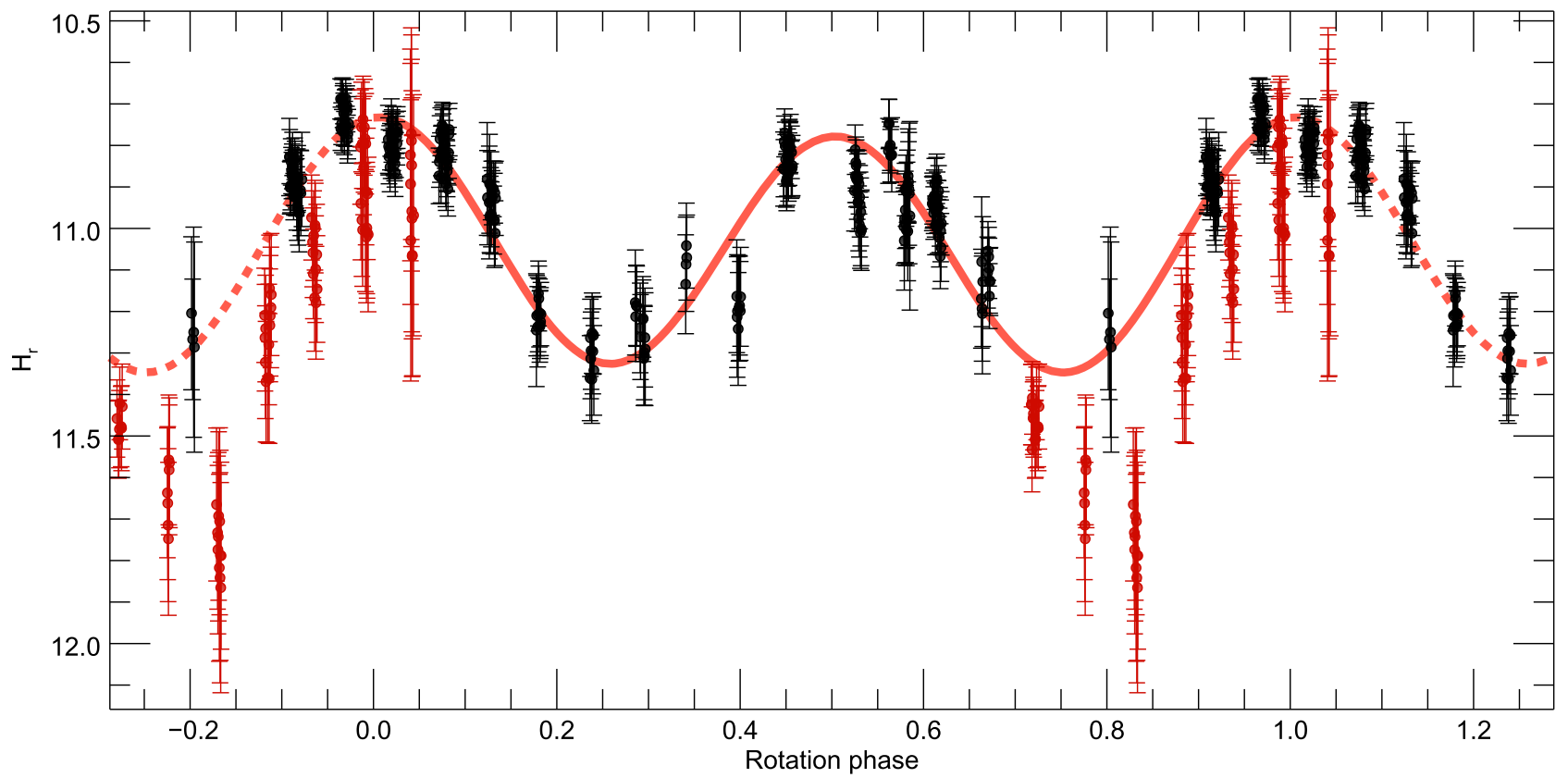

Figure 7. Phased 2013 light-curve data for Leucus from French et al. (2013). The solid curve is the 2-term Fourier fit to the 2016 data for a rotation period of $445.732 \mathrm{hr}$. These data are adjusted by a phase shift of 0.27 to compensate for the difference in viewing geometry between 2013 and 2016 . The red points were excluded from the full period fit.

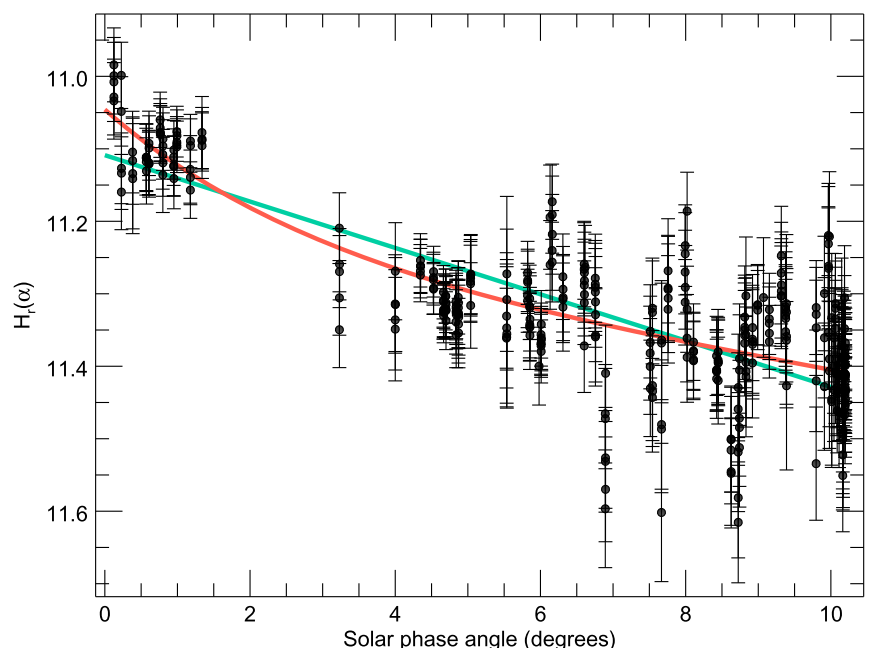

Figure 8. Leucus solar phase angle light curve. Distance corrections have been applied and the Fourier-fit light curve has been subtracted to leave the phase behavior. Only the data from this study are plotted here. The solid red curve shows the phase behavior for $G=0.575$. The solid green line is a linear phase fit. The data for this figure are provided as an electronic supplement to Figure 6.

essential to making sense of the prior light-curve results from French et al. (2013). The light curve is seen to have the same amplitude at the two epochs. The geometry shown in Figure 1 indicates that we have a vantage point roughly $90^{\circ}$ different between data taken in 2013 and 2016. The similarity in amplitude strongly suggests that the rotation axis is normal to our line of sight. If Leucus were also in a zero-inclination orbit, this would also indicate a low obliquity. However, Leucus has an inclination of $11^{\circ} .6$, meaning that its obliquity cannot be zero. With additional data, it will soon be easy to determine the pole direction. Regardless, this object should be carefully monitored for signs of a secondary object. The low obliquity will help somewhat in that search, but detection will be very

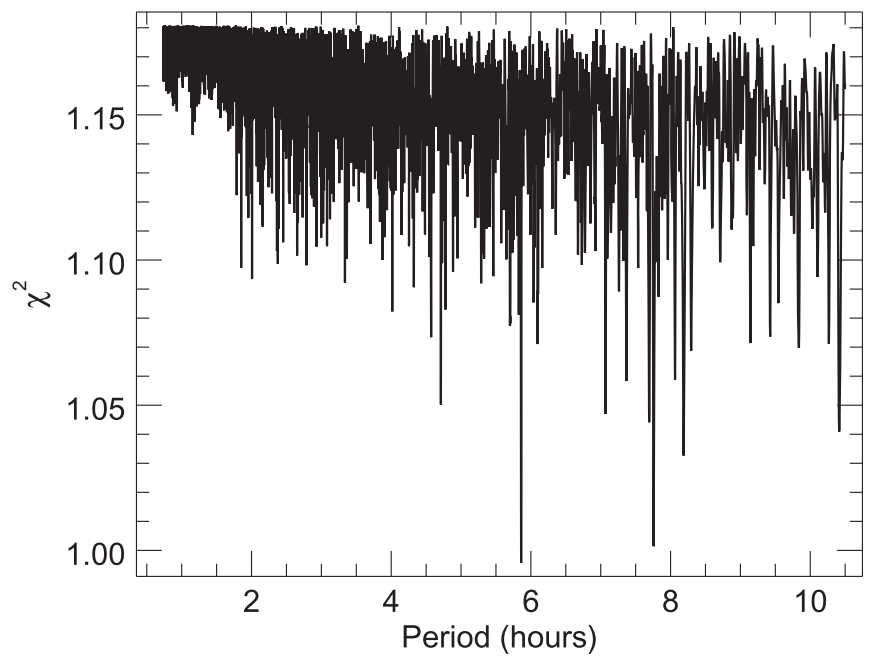

Figure 9. Period scan for Polymele. The second-best minimum is $41 \sigma$ away from the best minimum.

challenging if a secondary does not have the same orbit period as the rotation period of Leucus.

The light-curve amplitude gives us a constraint on shape for Leucus. Assuming a tri-axial shape, we can say $a \leqslant b<c$, where $a$ is the polar radius and is essentially unconstrained and $b$ and $c$ are the equatorial radii. An amplitude of $0.61 \mathrm{mag}$ translated to a constraint of $b / c=0.57$ which is a lower limit. If, as we suspect, the pole is normal to our line of sight then this limit is the actual ratio. If we assume the Grav et al. (2012) diameter of $34 \mathrm{~km}$ represents the mean size and $a=b$, then $b=26 \mathrm{~km}$ and $c=45 \mathrm{~km}$.

There are reports that the phase behavior of low-albedo objects, specifically Trojan asteroids, are significantly and systematically different compared to main-belt asteroids. French (1987) presented a phase curve of (1173) Anchises with a very shallow phase curve $\left(0.023 \mathrm{mag} /{ }^{\circ}\right)$ and 


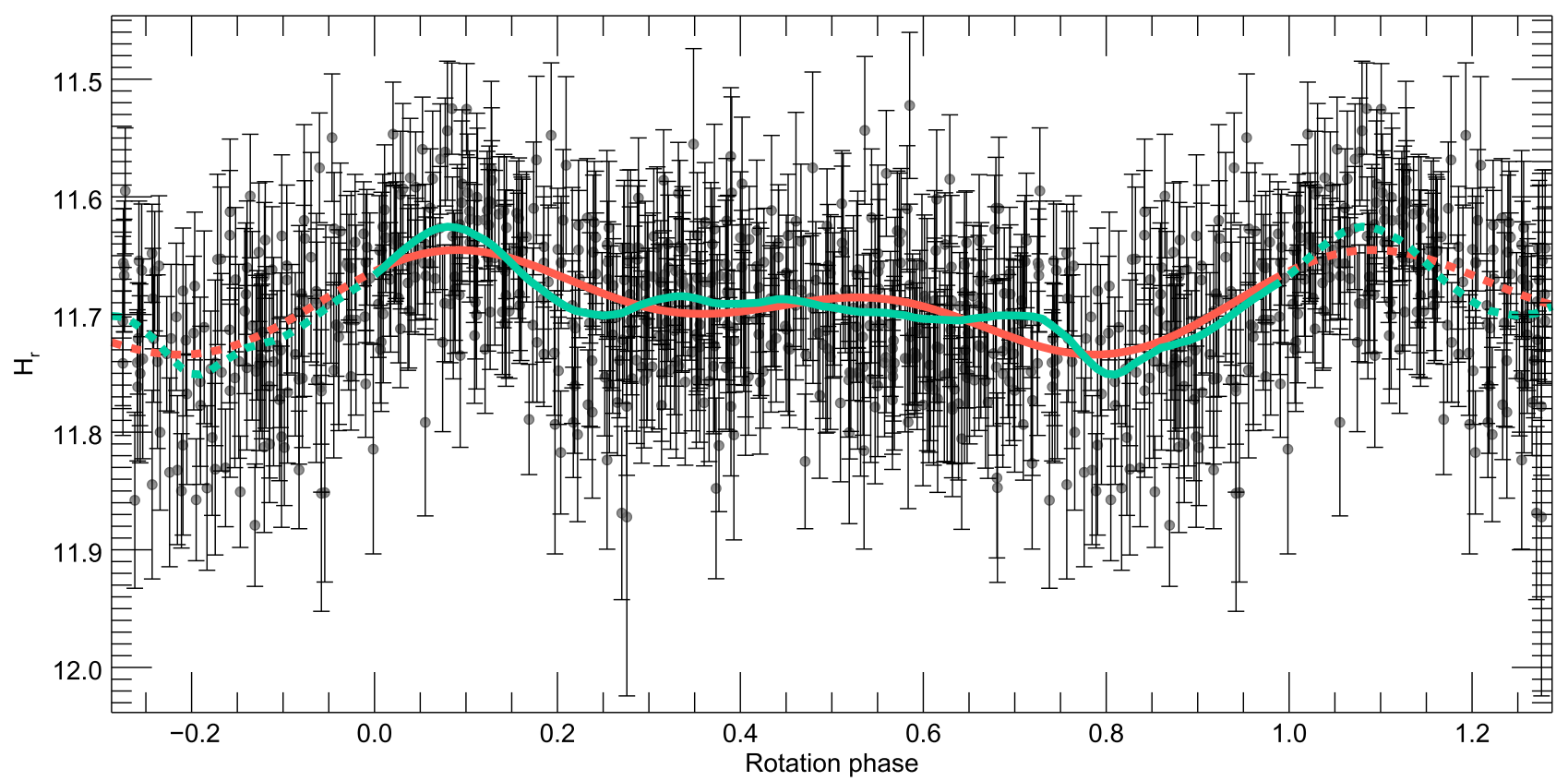

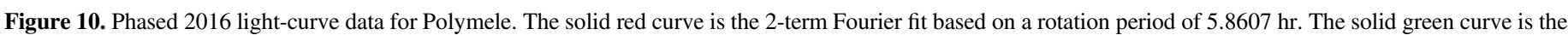

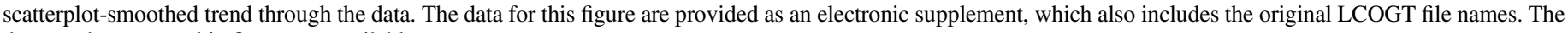
data used to create this figure are available.

Table 3

Light-curve Coefficients for Polymele

\begin{tabular}{ccccc}
\hline \hline$n$ & $a_{n}$ & $\sigma_{a}$ & $b_{n}$ & $\sigma_{b}$ \\
\hline 0 & 11.691 & 0.002 & $\ldots$ & $\ldots$ \\
1 & -0.023 & 0.003 & -0.014 & 0.003 \\
2 & -0.022 & 0.003 & +0.008 & 0.003 \\
\hline \multicolumn{5}{c}{$\chi^{2}=5.861$} \\
\\
& $|O-C|=0.05$ mag
\end{tabular}

Note. Parameters computed for a period of $5.8607 \mathrm{hr}$ and a phase coefficient of $G=0.22$. All observations taken with a SDSS $r^{\prime}$ filter.

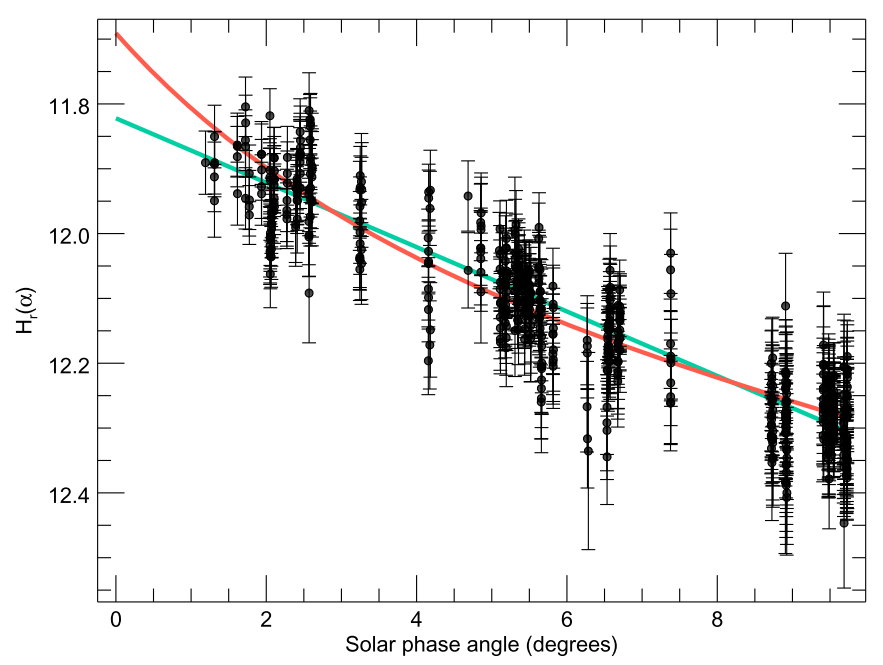

Figure 11. Polymele solar phase angle light curve. Distance corrections have been applied, and the Fourier-fit light curve has been subtracted to leave the phase behavior. The solid red curve shows the phase behavior for $G=0.22$. The solid green line is a linear phase fit. The data for this figure are provided as an electronic supplement to Figure 10.
$G=1.05 \pm 0.09$. In later work, Schaefer et al. (2010) and Shevchenko et al. (2012) demonstrated that Anchises is not alone in having a shallow linear solar phase variation. Objects like this suggest that a linear phase function could be better than the IAU $H, G$ photometric system. We cannot decide this issue with our data but simply point out that the Leucus photometry is better described with the standard $H G$ system. The strength of this conclusion rests entirely on having obtained data at a phase angle less than $1^{\circ}$. Had our data stopped at $2^{\circ}$, a linear phase function would appear to work just as well. For Polymele, this test is inconclusive, as we are missing the crucial low-phase data. In this case, a linear fit and an $H, G$ fit work equally well. Until these low-phase data are measured, the choice between phase function will have to be left open and thus the absolute magnitude for Polymele must also be considered more uncertain than our formal errors would indicate. Thus, our inferred albedo could be lower-the absolute magnitude for the linear case would indicate an albedo of $6.5 \%$. This is still higher than our result for Leucus but the difference is lower. In the end, there is a chance that these two objects could have the same low albedo. Additional data can help clarify any differences in albedo between these objects.

\section{Conclusions}

Our new observations provide new secure rotation periods for both Leucus and Polymele. Leucus is confirmed as having a very long rotation period and has a light-curve shape that bears some similarities to objects known or suspected to be binary (cf. Mann et al. 2007). Long rotation periods seem to be associated with binary objects, but we do not have any other data to confirm or refute the presence of secondary bodies. Our data show that the surface scattering properties of Leucus are a weaker function of phase angle $(G=0.575)$ than is typical for 
main-belt asteroids (Bowell et al. 1978) and quite different from the estimate from Vereš et al. (2015).

The lack of change in the light-curve amplitude and shape for Leucus between 2013 and 2016 (one-fourth of a revolution) suggests that the rotation pole is nearly normal to our line of sight. As the orbit of Leucus is not in the ecliptic, this rules out an obliquity of zero, but our data do suggest it is at least a lowobliquity object. Further observations are required to fully constrain the rotation pole but with a low-inclination view, it will be more difficult to get complete shape information. For now, we assume Leucus is a prolate ellipsoid but the minor axis of the body is as yet unconstrained. Stellar occultation observations will be essential for constraining the third dimension of its shape. Every six years, there will be enhanced odds for getting an occultation as Leucus crosses the galactic plane. Unfortunately, 2016 was one such year, so our next crossing will not be until 2022-after the launch of Lucy but before encounter.

If our suggestion of a low-inclination view of Leucus is correct, there will be ample opportunities to search for mutual events indicative of a secondary body. Further refinements in the rotation pole will be very helpful in narrowing down the search window. Clearly, high-S/N and high-cadence observations at minimum light would be very valuable for detecting any object that is in a tidally locked and synchronous orbit. With the significantly nonspherical shape, such configurations are even more likely.

Our understanding of Polymele is still very limited. Our new results indicate a low-amplitude light curve consistent with a nearly spherical object. Our determination of $G$ is also quite different from the estimate of Vereš et al. (2015). The spin rate is unremarkable, being neither near the spin barrier for an unconsolidated object nor very long (Pravec \& Harris 2000; Warner et al. 2009). The surface scattering properties, as revealed in the solar phase variation, have a typical value for asteroids, but we are missing phase angle data below $1^{\circ}$. Given that we only have one epoch of light-curve data, there are insufficient data to constrain the rotation pole orientation other than to suggest that if Polymele presented a pole-on configuration in 2016, the obliquity must be very high. Additional data will be needed to further investigate this object. If it is pole-on, the observing geometry will be different enough next year that a significant light curve could be visible if the object is non-spherical-as would be more typical for such a small body. As with Leucus, stellar occultations will also be valuable for a more complete shape determination. Objects this small have been typically very difficult to get with occultations, but the new Gaia astrometric catalog (Brown \& the Gaia Collaboration 2016; Mignard et al. 2016; Prusti \& The Gaia Collaboration 2016) will make this a viable endeavor.
This work makes use of observations from the LCOGT network.

Facility: LCOGT.

\section{ORCID iDs}

Marc W. Buie (iD https://orcid.org/0000-0003-0854-745X

\section{References}

Alard, C., \& Lupton, R. H. 1998, ApJ, 503, 325

Barucci, M. A., Cruikshank, D. P., Mottola, S., \& Lazzarin, M. 2002, in Asteroids III, ed. W. F. Bottke, Jr. et al. (Tucson, AZ: Univ. Arizona Press), 273

Bowell, E., Hapke, B., Domingue, D., et al. 1989, in Asteroids II, ed. R. P Binzel, T. Gehrels, \& M. S. Matthews (Tucson, AZ: Univ. Arizona Press), 524

Bowell, E. L. G., Chapman, C. R., Gradie, J. C., Morrison, D., \& Zellner, B. 1978, Icar, 35, 313

Brown, A. G. A. \& The Gaia Collaboration 2016, A\&A, 595, A2

Brown, T. M., Baliber, N., Bianco, F. B., et al. 2013, PASP, 125, 1031

Buie, M. W., \& Bus, S. J. 1992, Icar, 100, 288

Buie, M. W., Spencer, J. R., Porter, S. B., et al. 2018, AJ, submitted

Buie, M. W., Tholen, D. J., \& Wasserman, L. H. 1997, Icar, 125, 233

Bus, S. J., \& Binzel, R. P. 2002, Icar, 158, 146

Clark, B. E., Rivkin, A. S., Bus, S. J., \& Sanders, J. 2003, BAAS, 35, 955

French, L. M. 1987, Icar, 72, 325

French, L. M., Stephens, R. D., Coley, D., Wasserman, L. H., \& Sieben, J. 2015, Icar, 254, 1

French, L. M., Stephens, R. D., Coley, D. R., et al. 2013, MPBu, 40, 198

Grav, T., Mainzer, A. K., Bauer, J. M., Masiero, J. R., \& Nugent, C. R. 2012, ApJ, 759, 49

Hainaut, O. R., Boehnhardt, H., \& Protopapa, S. 2012, A\&A, 546, A115

Henden, A. A., Levince, S. E., Terrell, D., Smith, T. C., \& Welch, D. 2012, JAAVSO, 40, 430

Ivezić, Ž., Tabachnik, S., Rafikov, R., et al. 2001, AJ, 122, 2749

Levison, H. F. \& Lucy Science Team 2016, in 47th Lunar and Planetary Science Conf. (Houston, TX: LPI), 2061

Mann, R. K., Jewitt, D., \& Lacerda, P. 2007, AJ, 134, 1133

Mignard, F., Klioner, S., Lindegren, L., et al. 2016, A\&A, 595, A5

Miller, J. P., Pennypacker, C. R., \& White, G. L. 2008, PASP, 120, 449

Muinonen, K., Belskaya, I. N., Cellino, A., et al. 2010, Icar, 209, 542

Pravec, P., \& Harris, A. W. 2000, Icar, 148, 12

Prusti, T. \& The Gaia Collaboration 2016, A\&A, 595, A1

Schaefer, M. W., Schaefer, B. E., Rabinowitz, D. L., \& Tourtellotte, S. W. 2010, Icar, 207, 699

Shevchenko, V. G., Belskaya, I. N., Slyusarev, I. G., et al. 2012, Icar, 217, 202

Szabó, G. M., Pál, A., Kiss, C., et al. 2017, A\&A, 599, A44

Tedesco, E. F., Noah, P. V., Noah, M., \& Price, S. D. 2002, AJ, 123, 1056

Tholen, D. J. 1984, PhD thesis, Univ. Arizona

Vereš, P., Jedicke, R., Fitzsimmons, A., et al. 2015, Icar, 261, 34

Warner, B. D., Harris, A. W., \& Pravec, P. 2009, Icar, 202, 134

Yoshida, F., \& Nakamura, T. 2005, AJ, 130, 2900

Zacharias, N., Finch, C. T., Girard, T. M., et al. 2013, AJ, 145, 44

Zellner, B., Tholen, D. J., \& Tedesco, E. F. 1985, Icar, 61, 355 